\title{
A panacea for improving citizen behaviors? Introduction to the symposium on the use of social norms in public administration
}

\author{
Peter John", Michael Sanders", Jennifer Wang ${ }^{\dagger}$
}

\begin{abstract}
Recent years have seen a growth in the use of social norm messages by local and national governments across the world. These messages have primarily been used to induce desired behaviors among the noncompliant minority by pointing to the compliance of the majority. As well as being of considerable theoretical interest, these messages have a high level of empirical and experimental support in government settings as well as a few null and negative findings. In this introduction to the symposium, we offer an overview of research to date using social norms in public administration, reviewing what 'stylized facts' emerge, then introduce the articles included in the symposium.
\end{abstract}

$I^{\prime}$ $\mathrm{f}$ there is one lesson that has emerged from the large number of experimental interventions carried out by academics, businesses, and policy-makers in recent years, it is that publicizing a desired social norm encourages citizens to act. It seems enough for administrators to encode a message with the phrase 'nine of ten people do... [activity of interest]' and subsequently expect compliance and cooperation from citizens, with more people paying taxes, settling court fines, recycling their waste, and so on. In this way, descriptive norm messages have become a behavioral tool of choice for today's policy-makers. Indeed, applications of social norms are no doubt an important means through which to encourage desirable citizen behaviors, given that people are strongly influenced by what others do but may not always have accurate information about those behaviors. The popularity of social norms with policy-makers is not surprising, as it is a simple policy tool, easily deployed, and makes use of existing information. There is often an information asymmetry between citizens and administrators as administrators often have greater access

\footnotetext{
* King's College London

† Stanford University

Address correspondence to Peter John at

(peter.john@kcl.ac.uk)

Copyright: (C) 2019. The authors license this article under the terms of the Creative Commons Attribution 4.0 International License.
}

to information relative to citizens. The act of rectifying the asymmetry is inexpensive and can usually have beneficial effects as citizens can update their beliefs to reflect a reality that is often in the public interest.

In the light of this expansion of official and academic interest in social norms across a range of specialist fields and domains, and at the encouragement and sponsorship of the editors of JBPA, we decided to commission and edit this symposium on social norms in public administration, having a call for papers for a deadline of the end of June 2018. We cast the net wide in the specification:

\footnotetext{
"We are looking to invite submissions for a symposium on social norms (both descriptive and injunctive) in public administration, to be published in the Journal of Behavioral Public Administration (JBPA). Papers should provide evidence of novel applications of social norms, either in policy area, geography, or method of implementation. We envisage papers about taxes and fees, environmental behaviors, education, development outcomes, but we are not prescriptive and encourage application from any field. We particularly welcome papers that report null results from well-designed studies, and papers by serving civil servants and bureaucrats, as well as from academics and researchers."
} 
Although we encouraged those whom we knew already to respond to this call, we were pleased to find that most of the interest came from researchers from outside our networks, suggesting a larger field of scholars working in this area than we had imagined previously, which indicates the health and diversity of this research field. We hope that this collection of papers itself will increase the visibility of this area of research and encourage researchers using social norms in all kinds of contexts and with a range of methods and theoretical concerns to continue their work and encourage others to do so. We introduce these papers in the symposium in the coda of this introduction. Before we get to these summaries, we offer some more reflections on the theory behind the use of social norms. We place these papers in context by reporting a desk review of papers using descriptive social norms to illustrate themes and trends in the field.

\section{Theories of Social Norms}

There are two main forms of social norm-descriptive and injunctive. Following Cialdini et al. (1991), descriptive norms are those that "characterise the perception of what most people do" (p. 203), and injunctive norms are those that "characterise the perception of what most people approve or disapprove [of]" (p. 203). An example of a descriptive norm might therefore be a statement such as "nine out of ten people pay their tax on time", while an injunctive norm of the same type could be "nine out of ten people think that people should pay their tax on time". In the former, we determine something about the behavior of others; and in the latter, we learn something of their preferences.

Policy-makers are uniquely positioned to help correct public misperceptions about social norms, which may arise from over-reporting or biased recall by citizens of undesirable social acts and subsequent erroneous beliefs about the rate of actual misbehavior; or correct instances of undesirable norms arising out of pluralistic ignorance, when individuals respond to incentives to conform to what other people do, and believe that what they see of the behaviors of others reflects their true preferences (Bicchieri, 2005, p.15). Policy-makers can facilitate better-informed and more transparent communication with the public.

However, it is an open question as to whether or not citizens are consistently influenced by descriptive norms, and to what degree. Although there are many environments in which these theories have been tested, the targeted behaviors vary in terms of their desirability, are differentially easy to comply with, and are positioned against particular or competing reference groups that may be hard to anticipate. Moreover, it is possible that different ways of delivering descriptive social norms, given the type and direction of the desired behavior change, are more or less effective. Administrators thus need to know more about the parameters of this useful policy tool when advocating its use.

In the meantime, social norms interventions have become widely deployed and evaluated. The famous hotel towel study (Goldstein et al., 2008), whereby signs advertised a social norm that other hotel guests recycled their towels in an effort to encourage guests to recycle their own towels, has proven very popular, both in the popular press and in academic circles, with 2,127 citations in Google Scholar alone at time of writing. This study helped draw attention to the already developing field of social norms interventions, and prompted similar tests by both academics and by agencies on a range of activities, using randomized controlled trials and other robust methods. The large number of studies testing descriptive social norms across contexts makes it possible to begin developing better generalizations about the scope of this kind of intervention, while spurring further debate and work in this area.

\section{Study Selection}

We offer a broad, if not comprehensive, view of the work of field experimental, or randomized controlled trial, research on the public, administered either by academic researchers or by public administrators. Some fifty-one papers, drawn from governments and academic publications, are summarized and listed in the appendix. We have also taken pains to select studies that are relevant for public administrators. In practice this has meant studies that address outcomes that administrators could be potentially interested in, such as licensing, littering, electoral registration, and policy outcomes that they can directly regulate, such as electricity consumption as part of energy policy. It is not a systematic review, nor does it claim to be. In fact, it goes beyond the standard literature produced by database searches into the literature across public administration itself, including the grey literature produced by the now hundreds of government units applying behavioral science, which is so often concealed in its detail, and so varied in its content, 


\section{Figure 1}

\section{Distribution of Effect Sizes of Social Norm Interventions}

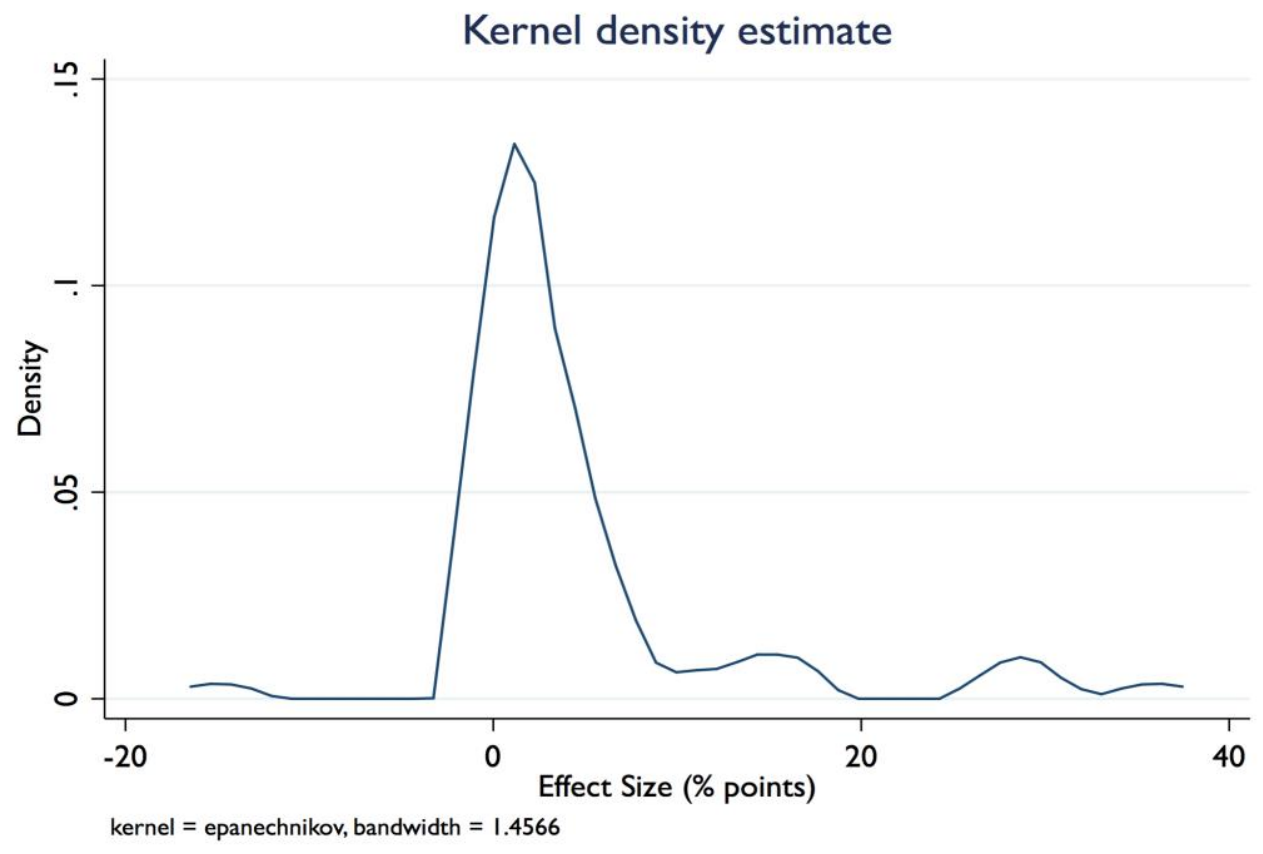

Figure 2

Distribution of Effect Sizes from Social Norms Interventions, Outliers Removed

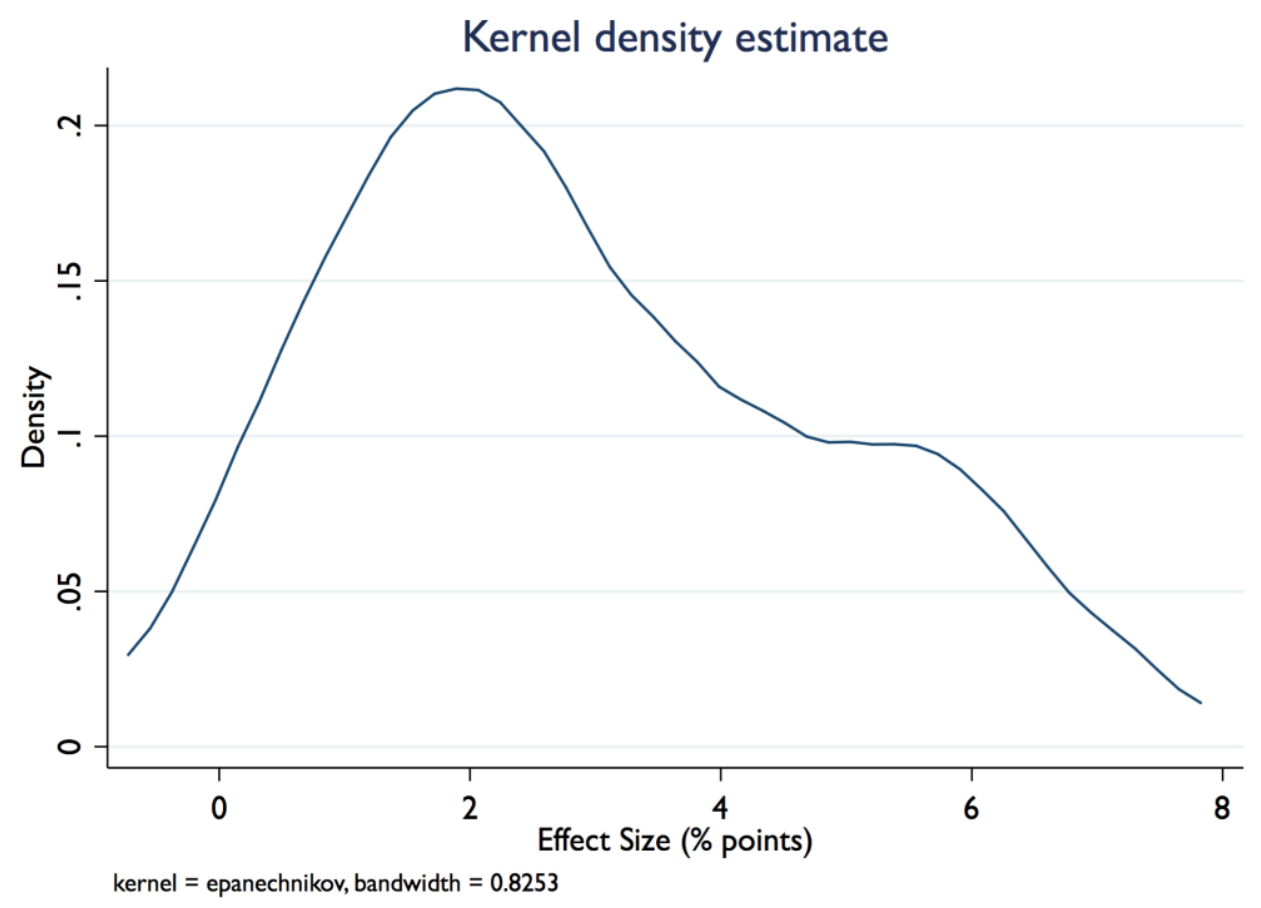


that it defies this kind of analysis. We aim to draw out themes from this literature and its associated 'dark matter', the unseen work of government units, partly to counter the widely documented phenomenon of unsuccessful experiments (by which we mean those that find null effects) being subject to "file drawing", whereby they are not written up for publication, and/or are not published if written up. In public administration, the risk of file drawing is acute and exacerbated by the specific context of public administration research. Randomized controlled trials conducted in government, often by government officials without formal consultation with university academics, can be both methodologically valid and produce interesting (and positive) results, but never find their way to publication simply because of the incentives of the officials who ran them. In particular, the job of a civil service researcher can be thought of as finding out "what works" and then implementing that result, not disseminating findings through peer review. Even among well-intentioned officials, the fact that publication of this kind is not a priority for their institution will lead to under-publication. In an attempt to redress this balance, our review of the literature includes studies conducted in a policy context that are unpublished or not yet available in a standard academic paper depository, such as SSRN.

\section{Findings}

In our analysis we review the findings of these studies that range across the fields of health, taxation, charitable giving, energy, environment (with a focus on recycling), voter turnout, and a few other single studies in areas such as licensing and consumer purchasing. The bulk of these studies are on taxation, energy, and the environment, and most are published. Overall, social norms appear to affect targeted outcomes in the desired direction. Only six studies did not show this effect with social norms, although eight other studies show norms only affecting one of the outcome variables of their study or one treatment arm. While our inclusion of unpublished or non-peer reviewed studies does not fully correct for publications bias, the unpublished work included in this review does not appear to differ in reporting that social norms worked on their outcome measure. Other kinds of selection bias are still a concern as there may be cases where norms do not work and would therefore not be put forward for publication or in an unpublished form except by personal enquiry; furthermore, there may be a group of studies where early negative results caused researchers to change topics or abandon the study before reporting any outcomes. However, it should be noted that many of the studies have a range of treatment conditions, which include other "behavioral" interventions tested alongside the descriptive norms, such as providing information. Although this 'multi-arm' approach taken by many government trials is the source of statistical concerns for many, it does have the effect of de-risking individual study arms and, all else equal, increasing the likelihood that a social norms null result will be published.

For a numerical comparison of effect sizes, we consider effect sizes in terms of percentage point changes in desired behaviors - where this is possible to determine based on the information reported and where the outcome measure is non-continuous - as is very often the case. In total this yields 63 effects across 43 of our 51 studies.

The main question to answer is whether there are differences in reported effect sizes across studies and whether these differences are attributable to differences in contexts such as intervention implementation and field of interest. The mean effect size observed is 4.5 percentage points, with a median effect size of 2.1 percentage points. Sixteen interventions report insignificant effects. We observe effect sizes ranging approximately 10 percentage points, with the low end at 1-2 percentage points, the middle at 2-5 percentage points, and the high end at 5-10 percentage points. There are very few (6) effect sizes greater than ten percentage points and the bulk fall in the middle range (see figures 1 and 2) which indicate that administrators should in general expect modest changes in behavior from this tool even if social norms appear to have an effect in most sectors of activities. Generally speaking, where there are citizen activities and a need for compliance, norms appear to play a universal role, at least for the locations and topics that have been studied so far.

The area that reports the largest and most consistent effects is within the sector of energy use. Effect sizes for these studies range from 2 to 10 per cent, backed by a relatively large number of studies. It would appear that the provision of a norm that is lower than a household's consumption is effective in reducing energy use, and it also appears that it is possible to counteract the boomerang effect of a high social norm increasing the consumption of low energy using households. Here we note that the norm provides information that is not available to households and gives them something attainable that they 
Figure 3

The Effect Sizes after Time

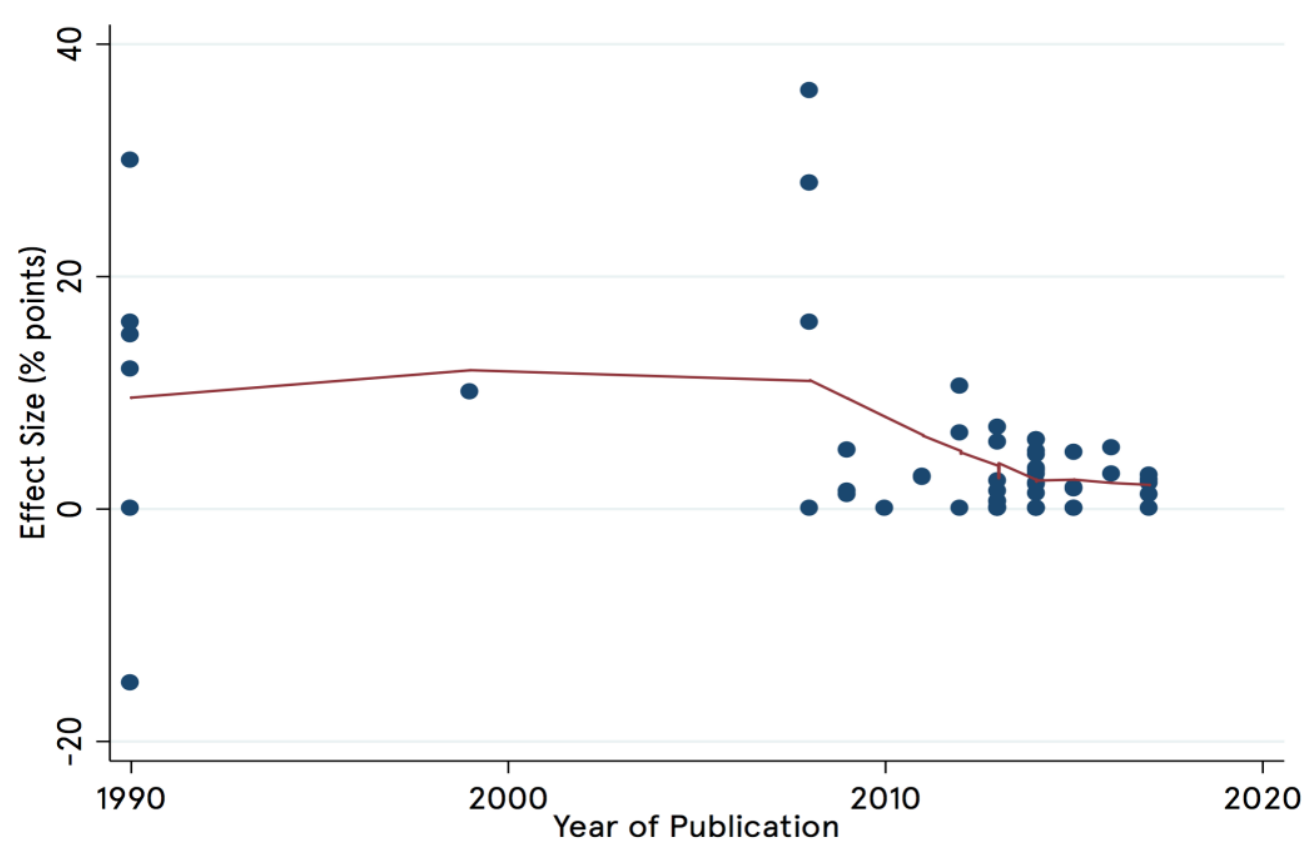

Figure 4

The Effect Sizes After Time, 2008 Onwards

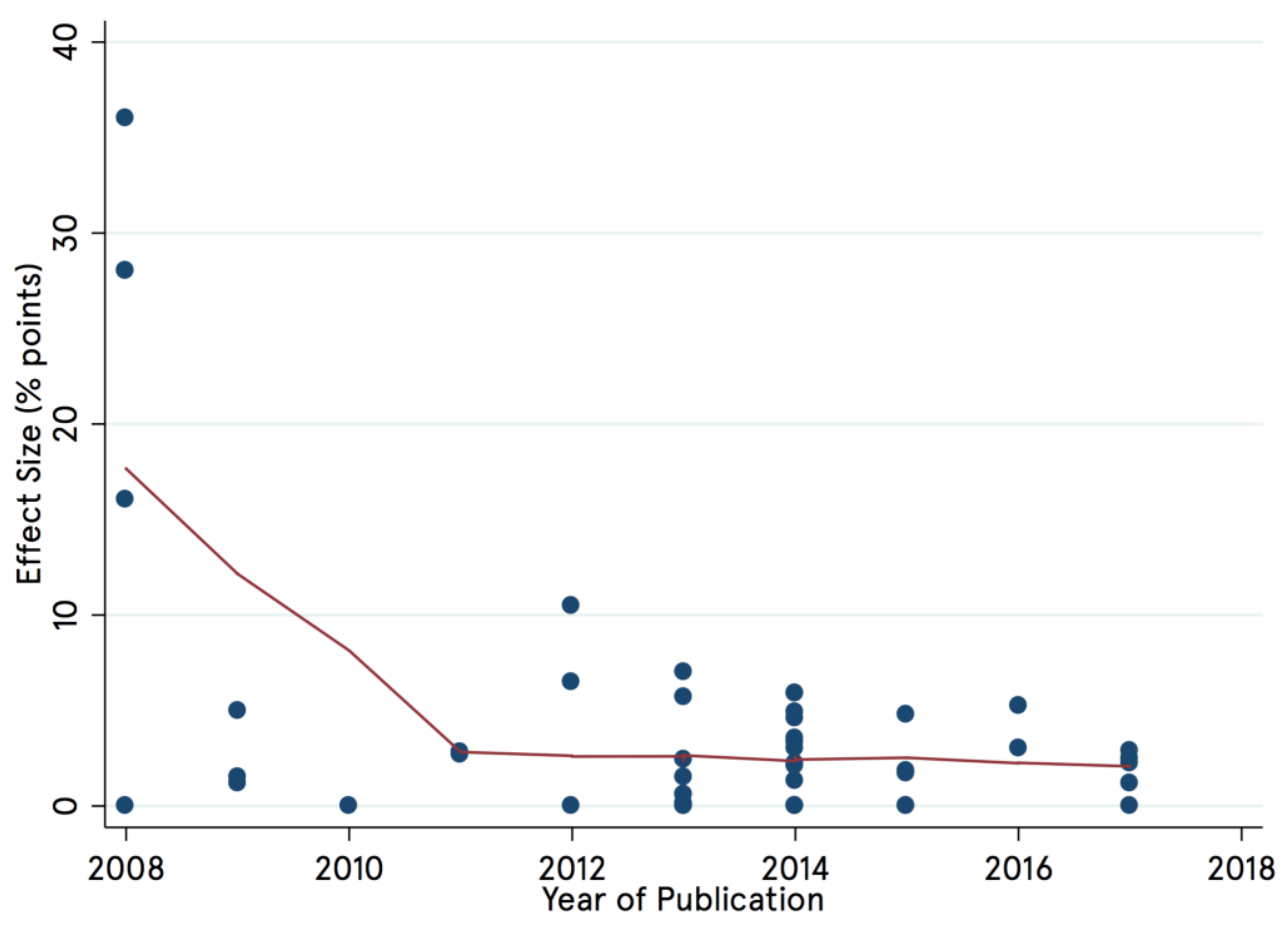


Figure 5

The Relationship Between Effect Size and Sample Size

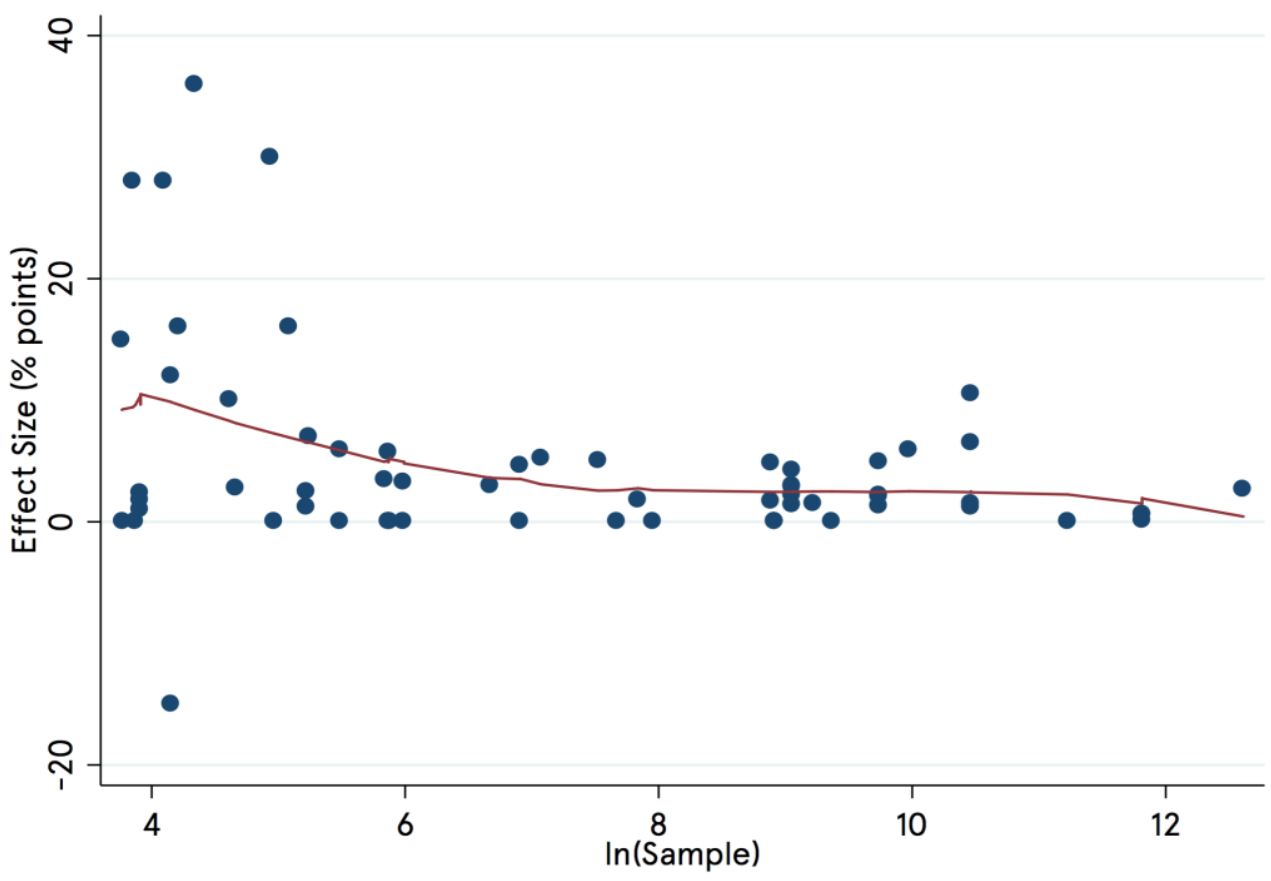

can also benefit from. In addition, there is some farfrom-conclusive evidence of a long-term effect of this norm (see Allcott \& Rogers, 2014). The need for further long-term studies of outcomes is not unique to social norms (Rogers \& Frey, 2014).

Of course, research is not a static endeavor, and so we should also be interested in changes in effect sizes reported over time. We present the results of this analysis in figures 3 and 4 . Figure 3 shows the effect sizes reported by each intervention over time (by year of publication), for all the studies where we have this information available. It is relatively hard to identify a trend in this data, as there are two discrete groupings of results, which can be thought of as "pre and post Nudge". Figure 4, therefore, presents this information only for studies from 2008 onwards.

We see some indicative evidence of a downward trend in effect sizes over time. It is not possible, however, to ascertain whether this decline is caused by changes in the areas studied, improvements in research methodology, or genuine declines in effect sizes.

The middle of these - improvements in methodology - can to a certain extent be investigated in our data. One factor commonly identified by scholars as a cause of the "replication crisis" in social psychology is that of small sample sizes in many social psychology studies. Although field experimentalism suffers less from this problem per se (the smallest per-cell sample size in our data is 44 participants, and the median is 1000), it also suffers more from noisy environments in which tests are carried out, and so a sample that might be sufficient for a lab experiment may not be so in field settings. There is also considerable heterogeneity in sample sizes in the experiments included in our study. Figure 5 shows the relationship between effect sizes and the natural $\log$ of per-cell sample size in our sample, again showing a downward trend.

\section{Summary of the Review}

We observe some differences in reported effect sizes by field, with interventions in more voluntary and active citizen arenas seeming to cause greater and more consistent changes in behavior, whereas interventions targeting more compulsory activities appear to have less of an effect. This may be a result of already high levels of cooperation and compliance in tax compliance, leaving slender margins for improvement. These differences aside, we note the consistency of these effects as a result of social norms interventions, and the relative absence of null and negative effects among our collated studies. Thus, it 
appears that social norms interventions are a consistent and reliable tool for policy-makers, consistent with the rising popularity of the use of this intervention by governments and policy-makers. It is important to recognize the diminishing effect of social norms interventions over time, and the smaller effects observed in larger scale studies. These factors should give us pause before declaring "case closed" on social norms as a policy intervention, and we argue that further research is needed to uncover the limitations of social norm use in public administration - something that is a key goal of the remainder of this symposium.

\section{Introduction to the Papers in the Symposium}

The papers we selected represent excellent examples of the emergent work in this field, though of course they are only a subset of the total output available and in progress.

The first article is on a vitally important topic, gender discrimination, which shows that social norms have come a long way from being thought to be mainly about more mundane issues like tax collection. Moreover, addressing discrimination is increasingly the focus of behavioral science and public interventions. In 'Descriptive norms and gender diversity: Reactance from men', Maliheh Paryavi, Iris Bohnet, and Alexandra van Geen deploy lab experiments to test whether the initial decision to hire proportions of male and female employees have effects on decisions down the line. These do not affect women, rather influencing men's decisions in reaction to it. This brings the important behavioral/psychological concept of reactance together with social norms, because it is important to see social norms operating dynamically over time as people react to changing contexts. The results from this experiment show how the way in which social norms are set influences their impact down the line, as well as showing the heterogeneity of the impact of social norms, another important and neglected topic of research. It also shows that the descriptive and injunctive can at times be present in the same intervention. We expect this piece to stimulate a line of further research, hopefully with experiments done in the field.

Of course, researchers should keep focusing on the classic tax collection domain as this field can yield new findings and provide innovative twists, as the next papers achieves. Christopher Larkin, Michael Sanders, Isabelle Andresen, and Felicity Algate in
'Testing local descriptive norms and salience of enforcement action: A field experiment to increase tax collection' address the important issue of what mechanism drives citizens to respond to tax collection efforts. It has always been known the audit and enforcement remain important tools for tax collection with the nudge-style interventions complementing rather than replacing the more traditional tools (e.g. Kirchler et al., 2007). But we do not know how these mechanisms work especially in comparison to each other in the same study design. The design for this paper does just this, reporting a field experiment with an English local authority's tax collection procedures, and shows how the social norm outperforms enforcement strategy, even though both work well. It is an important addition to the long line of studies that are contained in our desk review. Future work might wish to interact these treatments to understand how they might work in combination with each other and as substitutes.

The next example shows how social norms are an important concept in understanding health outcomes and prevention strategies, which is a large area of academic and policy activity. In 'Parents' social norms and children's exposure to three behavioral risk factors for chronic disease', Olivier Drouin, Jonathan P. Winickoff, and Anne N. Thorndike show how the behavior of parents toward their children is linked to social norms. This relates to the classic finding in many studies that people do not correctly calculate or perceive the behavior of others, which is also the basis of the effectiveness of social norm interventions. This misperception in this controversial field is an important first step, which justifies further research testing interventions to change the perception of social norms, possibly in an experimental set up (if the ethical sensitivities can be addressed).

Showing again the diversity of the fields covered even in a short symposium, Matt Biggar, in 'Unpacking the influence of social norms and past experience on commute mode choice', makes a contribution to the large field of transport studies, as well as showing that qualitative methods are as important as experiments and statistical analyses in offering insights and knowledge about how social norms work. Biggar matches individuals to help generate valid inferences about the influence on social norms. The research shows that prior experience and social networks influence social norms powerfully, affecting how people make transport choices in ways that move beyond the more traditional cost-benefit approach. As with 
all the papers in this symposium, there are implications for future research testing interventions to modify those social norms, and for policy-makers in designing them.

Finally, Jessica Leight and Elana Safran in 'Increasing immunization compliance among schools and day care centers: Evidence from a randomized controlled trial' test for norm compliance over immunization in a sample of 700 schools. This study is impressive in its application to a large spatial area, covering both public health and education, and targeted to organizations. The null results contribute the main objective of our review and of this symposium, that of publishing the full range of studies of social norms regardless of direction of findings.
Overall, the contributions to the symposium shows what a large field the study of social norms in public administration settings has become, as well as showing the diversity of topics covered. Moreover, each makes a contribution in a substantively important area for policy-makers, whether it is general discrimination, ensuring a flow of revenues to fund public services, affecting the health of children, or in achieving environmental goals of using more diverse and sustainable forms of transport. With this dynamism and policy relevance, we expect there will be many more social norm studies appearing in the years to come.

\section{References}

Bicchieri, C. (2005). The Grammar of Society: The Nature and Dynamics of Social Norms. New York: Cambridge University Press. Chapter 5, 176-208.

Cialdini, R. B., Kallgren, C. A., \& Reno, R. R. (1991). A focus theory of normative conduct: A theoretical refinement and reevaluation of the role of norms in human behavior. In: Berkowitz, L., Ed., Advances in Experimental Social Psychology, Academic Press: San Diego, Vol. 24, 201-234.

Goldstein, N. J., Cialdini, R. B., \& Griskevicius, V. (2008). A room with a viewpoint: Using social norms to Motivate environmental conservation in hotels. Journal of Consumer Research. 35: 472-482.

Kirchler, E, Muehlbacher, S, Kastlunger, B, \& Wahl, I (2007). Why pay taxes? A review of tax compliance decisions. International Studies Program Working Paper, 7-30, Georgia State University. 